OPEN ACCESS

Edited by:

Manish A. Shah,

Cornell University, United States

Reviewed by:

Jaafar Bennouna,

Centre Hospitalier Universitaire

(CHU) de Nantes, France

Rachna Shroff,

University of Arizona, United States

*Correspondence:

Wei He

hewei726@zzu.edu.cn

${ }^{t}$ These authors have contributed equally to this work

Specialty section: This article was submitted to Cancer Molecular

Targets and Therapeutics, a section of the journal

Frontiers in Oncology

Received: 27 January 2021 Accepted: 12 April 2021

Published: 03 May 2021

Citation:

Zhang G, Gong S, Pang L, Hou L and He W (2021) Efficacy and Safety of Apatinib Treatment for Advanced Cholangiocarcinoma After Failed Gemcitabine-Based Chemotherapy: An Open-Label Phase II Prospective Study. Front. Oncol. 11:659217. doi: 10.3389/fonc.2021.659217

\section{Efficacy and Safety of Apatinib Treatment for Advanced Cholangiocarcinoma After Failed Gemcitabine-Based Chemotherapy: An Open-Label Phase II Prospective Study}

\author{
Ge Zhang ${ }^{\dagger}$, Shuai Gong ${ }^{\dagger}$, Lina Pang, Lixia Hou and Wei He* \\ Department of Oncology, The First Affiliated Hospital of Zhengzhou University, Zhengzhou, China
}

Purpose: As a novel small-molecule vascular endothelial growth factor receptor-2 tyrosine kinase inhibitor (VEGFR2-TKI), Methylsulfonic apatinib (apatinib) exhibits a specific antitumor effect in various solid tumors via inhibition of angiogenesis. The present study was performed to evaluate the clinical efficacy and safety of apatinib in the treatment of advanced cholangiocarcinoma after failed gemcitabine-based chemotherapy.

Patients and Methods: This was a prospective open-label phase II trial (NCT03521219). A total of 32 patients, in whom gemcitabine-based first-line chemotherapy for advanced intrahepatic cholangiocarcinoma had failed, were consecutively enrolled in a prospective, open, exploratory, and single-center clinical trial from November 2017 to November 2018. They were treated with apatinib mesylate second-line monotherapy (orally, $500 \mathrm{mg}$ per day for a cycle of 28 days) until progressive disease or unacceptable toxicity. Using Response Evaluation Criteria in Solid Tumor version 1.1 (RECIST 1.1) and the Common Terminology Criteria for Adverse Events version 4.0 ( $\mathrm{NCl}$-CTCAE 4.0), the efficacy and adverse were evaluated, respectively. Kaplan-Meier method was used for survival analysis.

Results: Twenty-six patients were enrolled in full analysis set. At the end of follow-up, two patients were lost to follow-up, 24 of 26 patients in FAS were included in efficacy analyses. For the efficacy analysis set, the objective response rate (ORR) was 20.8\% [95\% confidence interval (Cl): 9.24-40.47\%] and the disease control rate (DCR) was $62.5 \%$ (95\% Cl: 112.86-387.14 days). One patient (4\%) showed complete response (CR), 4 patients (17\%) showed partial response (PR), 10 patients (41.7\%) stable disease (SD), and 9 patients (37.5\%) had progressive disease (PD). Meanwhile, apatinib therapy achieved the median progression-free survival PFS was 95 days (95\% Cl: 79.70-154.34 days), and the median OS was 250 days (95\% Cl: 112.86-387.14 days). Furthermore, univariate analysis revealed that age and tumor's anatomic location significantly affected PFS $(\mathrm{P}<$ 
0.05). The most common clinically adverse events (AEs) included myelosuppression (69.2\%), hypertension (57.7\%), proteinuria (46.2\%). The AEs were mild, mainly in grade 1 or 2, and no toxicity-induced death occurred.

Conclusion: Apatinib monotherapy is an effective and promising regimen for treating patients with advanced cholangiocarcinoma who experienced failure of gemcitabinebased chemotherapy.

Keywords: apatinib, advanced cholangiocarcinoma, prospective study, efficacy, angiogenesis

\section{INTRODUCTION}

Cholangiocarcinoma is a highly malignant tumor with poor prognosis. Based on the anatomic site, it is divided to intrahepatic and extrahepatic cholangiocarcinoma. The morbidity and mortality rates are high in patients aged 30 to 50 years and have been increasing recently (1). Due to insidious onset, non-specific symptoms of early cholangiocarcinoma, and lack of a particularly satisfying marker or imaging technique for diagnosis $(2,3)$, many cases are already in the middle-late or advanced stage at the time of the treatment, and thus, only suitable for systemic or palliative therapy. Considering that cholangiocarcinoma shows insensitivity to radiochemotherapy, surgical resection is the only means of radical therapy. However, only $10 \%$ of patients diagnosed at the early stage are eligible for surgical resection, which is why 5 -year survival rate is very poor (only 5\%) (4). However, although gemcitabine combined with cisplatin or 5-fluorouracil as the "gold standard" for first-line treatment has been confirmed to improve survival, the median OS time is still shorter than 1 year. Given that there is no standard second-line treatment regimen at present, it is important to urgently establish novel therapeutic methods to improve survival time and achieve low toxicity.

Recently, anti-angiogenic therapies have shown promising results. Namely, angiogenesis plays a significant role in tumor growth, metastasis, and recurrence; thus, anti-angiogenic therapy has great potential for cancer therapy (5). Considering sustained overproduction of VEGF owing to the hypoxic environment in the tumor after chemotherapy, VEGF is likely the fundamental factor promoting angiogenesis by VEGFR-mediated pathways (6). VEGFR-2, highly expressed on vascular endothelial cells and prominently mediating VEGF's angiogenic efficacy, has become the critical target of anti-angiogenesis therapy $(7,8)$.

Methylsulfonic apatinib (Jiangsu Hengrui Medicine Co.,Ltd, Jiangsu, China), hereinafter referred to as apatinib, an antiangiogenic drug with significant antineoplastic activity, was developed independently in China and approved by the China State Food and Drug Administration (CFDA) for subsequent-line therapy of advanced gastric or gastroesophageal junction adenocarcinoma (9). As a small-molecule tyrosine kinase inhibitor selectively targeting VEGFR-2, apatinib inhibits endothelial cell proliferation, reduces tumor microvascular density, and promotes cell apoptosis via downregulation of the VEGF pathways to achieve suppression of tumor growth and recurrence $(10,11)$. Apatinib has been applied for various types of malignancies and exerted obvious survival benefit with tolerable toxicity. It was specially approved as the third-line therapy in patients with advanced or metastatic chemorefractory gastric cancer $(12,13)$. However, there has been no definite conclusion concerning its efficacy and clinical safety for advanced cholangiocarcinoma. Here, we report an open-label phase II trial (ClinicalTrials.gov: NCT03521219) to conduct a prospective evaluation of the therapeutic effect and safety of apatinib in the second-line treatment of advanced cholangiocarcinoma.

\section{PATIENTS AND METHODS}

\section{Patients' Eligibility}

The inclusion criteria were as follows: 1 . Male and female aged 1875; 2. Eastern Cooperative Oncology Group Performance Status (ECOG) score of $0-2$ points; 3 . Histological or cytological diagnosis of recurrent or metastatic advanced cholangiocarcinoma after failure or intolerance of gemcitabine-based first-line chemotherapy regimens; 4. At least one measurable lesion by imaging examination [computed tomography (CT) or magnetic resonance imaging $(\mathrm{MRI}) \geq 10 \mathrm{~mm}$; CT scan thickness not greater than $5 \mathrm{~mm}$ ], and no radiotherapy or other local therapy performed unless progression after treatment occurred (RECIST 1.1); 5. Expected life expectancy $\geq 12$ weeks; 6 . Acceptable function of vital organs: absolute neutrophil count $\left(\right.$ ANC) $\geq 1.5 \times 10^{9} / \mathrm{L}$; platelet count $\geq 75 \times 10^{9} / \mathrm{L}$; hemoglobin $\geq 8 \mathrm{~g} / \mathrm{dl}$; serum proteins $\geq 2.8 \mathrm{~g} / \mathrm{dl}$; serum total bilirubin $\leq 3$ times upper limit of normal value (ULN), and ALT and AST $\leq 2.5$ times ULN (if liver metastasis is present, ALT and AST $\leq 5$ times ULN); creatinine clearance $\geq 50 \mathrm{ml} / \mathrm{min} ; 7$. No serious drug allergy history; 8 . Subjects volunteered to participate in the study. Prior to procedures, informed consent was signed by each patient who have satisfactory compliance and can cooperate with follow-up.

The exclusion criteria were as follows: 1. Patients who had undergone targeted therapy; 2 . Contraindications including active hemorrhage, ulcers, intestinal perforation, intestinal obstruction, within 30 days of major surgery, hypertension that cannot be controlled by drugs, cardiac insufficiency III or IV, and severe dysfunction of the lungs and the kidneys; 3. Coagulation disorders [international normalized ratio (INR) $>1.5$, thrombin time $(\mathrm{PT})>$ $\mathrm{ULN}+4 \mathrm{~s}$, or activated partial thromboplastin time (APTT) $>1.5$ ULN), hemorrhagic tendency, or undergoing thrombolytic therapy or anticoagulant therapy; 4 . Routine urinalysis suggested urinary protein $\geq++$ or 24 -h urinary protein excretion $\geq 1.0 \mathrm{~g} ; 5$. 
Pregnancy and lactation; 6. Other malignancies that had been diagnosed within 5 years prior to the first use of the study drugs, except squamous cell carcinoma or basal cell carcinoma that had been effectively treated and/or carcinoma in situ of the cervix or breast carcinoma that had been effectively removed; 7. Other situations that may influence the conduct and outcome of the clinical research. The quitting criteria were as follows: 1 . Patients who could not be treated according to the study protocol; 2 . Patients who asked to quit; 3. Patients who were not fit to continue the treatment.

The approval of this study was obtained by the First Affiliated Hospital of Zhengzhou University Ethic Committee.

\section{Treatment}

All of the patients were treated with apatinib mesylate at $500 \mathrm{mg}$ orally daily, administered half an hour after the meal, for a cycle of 28 days until significant disease progression, drug intolerance, or patients' decision. Grades 3-4 drug-related adverse events resulted in dosage reduction to $250 \mathrm{mg}$ per day or interruption for several days until symptoms resolved to grade 1-2 and stabilized. Symptomatic treatments were applied with or without modification of doses for the management of toxicities during the procedure.

The comprehensive medical history and clinical and laboratory data were recorded at the beginning of the treatment. Qualified subjects entered the treatment stage after a baseline evaluation. The patients underwent CT or MRI to radiographically evaluate tumor's response to treatment every 7 to 9 weeks during maintenance treatment, including objective tumor location and size, perineural invasion, lymphovascular invasion, and adverse events were collected. Moreover, performance status, blood pressure, blood routine parameters, urine, liver and kidney function, and electrolytes were monitored every 2 weeks.

All of the patients were followed up for 90 days to assess tumor recurrence after the last medication, undergoing a physical examination, blood testing, CT, or MRI at follow-up every 8 weeks ( \pm 7 days) until disease progression, initiation of a new therapy, or death for the subjects out because of non-PD. For subjects out because of PD and those non-PD subjects completing the follow-up, a survival follow-up was carried out once per month. The enrolled patients were followed up regularly, and the drugs' compliance and adverse events were assessed. During the follow-up, our trained clinical physicians would call them for updates (if they could not go to the hospital considering poor health following treatment).

\section{Efficacy and Safety Assessments}

Response Evaluation Criteria in Solid Tumor version 1.1 (RECIST 1.1) criteria were used for efficacy evaluation. The patients were categorized into four groups according to tumor response: complete response (CR), partial response (PR), disease stabilization ( $\mathrm{SD}$ ), and disease progression (PD). The primary endpoint was objective remission rate (ORR), while the secondary endpoints were multiple, including the assessment progression-free survival (PFS), overall survival (OS), and disease control rate (DCR). ORR was determined as the sum of CR and $\mathrm{PR}$, while DCR was calculated as the sum of the CR, PR, and SD.
PFS referred to the time from the initiation of treatment with apatinib to the time of disease progression confirmed radiologically or the end of follow-up, whichever occurred first. OS was defined as the time interval between the initiation of the first treatment until death or the last follow-up date. Adverse events (AEs) were in accordance with the frequency and severity of toxicities, reported and graded by investigators on the basis of the Common Terminology Criteria for Adverse Events version 4.0 (CTCAE 4.0).

\section{Data Analysis}

Our primary endpoint was ORR. In the setting of the initial sample size, a systematic review of Lamarca A et al. reported that the weighted mean ORR was 7.7\% (95\% CI: 4.6-10.9\%) in the 25 studies that evaluated the use of second-line chemotherapy for advanced cholangiocarcinoma patients (including 14 phase II clinical trials, 9 retrospective analyses, and 2 case reports) (14). Under this minimum value, treatment would be considered as treatment failure. We estimated the sample size as 25 patients under an expected ORR of $20 \%$ and a minimal efficacy of $7.7 \%$ by using PASS 19. This design provided an alpha error of $\alpha=0.05$ (two-sided) and a beta error of $\beta=0.2$ ( $80 \%$ power). Considering the leakage rate of $20 \%$, we decided to enroll a total of 32 patients. All participants receiving at least one dose of apatinib were included for the analysis.

Statistical analysis was performed using the SPSS software version 19.0 (IBM, Chicago, IL, USA). Continuous variables were expressed as median (25-75th percentiles), and categorical variables were expressed as percentage (\%) or number. The 95\% confidence interval (CI) for the primary endpoint ORR were calculated using the Clopper-Pearson method. KaplanMeier method was used for survival analysis to determine median PFS and OS, with 95\% CI, and the survival curve was calculated. Log- rank test was used to analyze factors affecting survival benefit. The $\mathrm{P}$-value $<0.05$ was considered to be statistically significant.

\section{RESULTS}

\section{Patients' Characteristics}

From November 2017 to November 2018, a total of 32 patients with advanced cholangiocarcinoma were enrolled in our study and underwent apatinib therapy in the First Affiliated Hospital of Zhengzhou University. Thirty-two enrolled patients with advanced cholangiocarcinoma signed informed consent at the time we initiated this study, while six patients of them were excluded for withdrawing the consents prior to the first dose of apatinib. Ultimately, 26 patients were included in full analysis set (FAS).

Among the 26 patients included, 14 (53.8\%) were men and 12 (46.1\%) were women. The median age was 58 years (range: $28-$ 78 years). ECOG 0 or 1 was present in most cases $(80.8 \%)$. Twelve patients (46.2\%) were diagnosed as intrahepatic cholangiocarcinoma, while 14 patients (53.8\%) had extrahepatic cholangiocarcinoma. The therapy protocols were determined based on the patient's general status, weight, age, and 
tolerance. Surgical resection could not be a choice in any of the 26 patients. All of the patients experienced progression after gemcitabine-based first-line chemotherapy regimens. They were prescribed with second-line monotherapy (apatinib with an initial dose of $500 \mathrm{mg}$ ). The most common metastasis location was liver $(100.0 \%)$, followed by lungs $(61.5 \%)$ and celiac lymph nodes $(61.5 \%)$. Before treatment with apatinib, previous therapy was accepted by 26 patients, including combination cisplatin plus gemcitabine $(n=10)$, gemcitabine plus capecitabine $(n=7)$, gemcitabine plus fluoropyrimidine $(n=5)$, and gemcitabine with oxaliplatin $(n=4)$. The median duration of prior gemcitabinebased therapy was $4.71 \pm 2.32$ months (range: 1.4-8.6 months). Patient baseline characteristics at the initiation of treatment are summarized (Table 1).

\section{Efficacy of Apatinib Treatment}

A total of 26 patients received at least one cycle of apatinib. The follow-up continued until all the patients met PFS and OS, and the median duration of follow-up was 8.3 (range: 0.9-28.0) months. Of 26 patients, 2 were lost to follow-up, 1 did not attend the follow-up visit due to a lack of time, and 1 stopped using apatinib and dropped out for another reason. At the end of follow-up, 24 of 26 patients in FAS were included in efficacy analyses, available for assessment of efficacy, according to investigator assessment of targeted lesions using CT or MRI.

TABLE 1 | The baseline characteristics of 26 patients.

\begin{tabular}{|c|c|c|}
\hline Characteristics & $\mathbf{N}$ & Percentage (\%) \\
\hline \multicolumn{3}{|l|}{ Sex } \\
\hline Men & 14 & 53.8 \\
\hline Women & 12 & 46.1 \\
\hline \multicolumn{3}{|l|}{ Age (years) } \\
\hline$\leq 60$ & 18 & 69.2 \\
\hline$>60$ & 8 & 30.8 \\
\hline \multicolumn{3}{|l|}{ ECOG performance status } \\
\hline 0 & 9 & 34.6 \\
\hline 1 & 12 & 46.2 \\
\hline 2 & 5 & 19.2 \\
\hline \multicolumn{3}{|l|}{ CA199 (U/ml) } \\
\hline$\leq 37$ & 10 & 38.5 \\
\hline$>37$ & 16 & 31.5 \\
\hline \multicolumn{3}{|l|}{ AFP (ng/ml) } \\
\hline$\leq 400$ & 7 & 26.9 \\
\hline$>400$ & 19 & 73.1 \\
\hline \multicolumn{3}{|l|}{ Anatomic location of the tumor } \\
\hline Intrahepatic & 12 & 46.2 \\
\hline Extrahepatic & 14 & 53.8 \\
\hline \multicolumn{3}{|l|}{ Metastasis } \\
\hline Liver & 26 & 100.0 \\
\hline Lungs & 16 & 61.5 \\
\hline Celiac lymph nodes & 16 & 61.5 \\
\hline Pancreas & 4 & 15.4 \\
\hline Adrenal gland & 3 & 11.5 \\
\hline \multicolumn{3}{|l|}{ Previous therapy } \\
\hline Gemcitabine plus cisplatin & 10 & 38.5 \\
\hline Gemcitabine plus capecitabine & 7 & 26.9 \\
\hline Gemcitabine plus fluoropyrimidine & 5 & 19.2 \\
\hline Gemcitabine plus oxaliplatin & 4 & 15.4 \\
\hline
\end{tabular}

$N$, the number of patients; ECOG, Eastern Cooperative Oncology Group.
Short-term curative effect: The evaluation of the best response was shown in accordance with RECIST v1.1. as follows: 1 patient (4.16\%) showed CR, 4 patients (16.67\%) showed PR, 10 patients (41.67\%) had SD, and 9 patients (37.50\%) had PD. The ORR was $20.8 \%$ (95\% CI: 9.24-40.47\%), and the DCR was $62.5 \%$ (95\% CI: 42.71-78.84\%). Long-term efficacy: Long-term curative effect of apatinib was analyzed by the Kaplan-Meier method for PFS and OS. The median PFS was 95 days [95\% confidence interval (CI): 79.70-154.34 days], and the median OS was 250 days (95\% CI: 112.86-387.14 days). Kaplan-Meier survival curves for PFS and OS are shown in Figure 1.

Univariate analysis revealed that some univariate factors affected the patients' survival (Table 2). There was no statistically significant effect of sex. However, age was a significant independent factor correlated with PFS. The median PFS was significantly improved from 49 days (95\% CI: 2.8-95.2 days) in the patients age $>60$ years old, to 127 days (95\% CI: 64.3-189.7 days) in the patients age $\leq 60$ years old ( $P=0.010$, Figure 2$)$. We showed that the tumor's anatomic location was associated with survival benefits $(P=0.08$, Figure 3). The median PFS was 153 days (95\% CI: 79.7-226.3 days) for intrahepatic cholangiocarcinoma, which was significantly longer than extrahepatic cholangiocarcinoma (72 days, 95\% CI: 21.3-221.7 days). ECOG performance status and CA199 were not linked to survival benefit $(\mathrm{P}>0.05)$.

\section{Safety}

The safety analysis set included all 26 patients. The most frequent adverse effects are listed (Table 3). We observed that the majority of toxicity considered to be associated with apatinib treatment was mild, mainly classified as grade 1 or 2 . One of the most common toxicities was bone marrow suppression (18/26, 69.2\%), manifested as leukocytopenia and thrombocytopenia. Fifteen patients $(57.7 \%)$ had secondary hypertension and 12 patients (46.2\%) developed proteinuria. Hand-foot syndrome occurred in 10 patients (38.5\%). Seven patients (26.9\%) had fatigue, and in seven patients $(26.9 \%)$ anemia was found. Liver dysfunction was observed during the treatment in some patients, including elevated transaminase $(7 / 26$, 26.9\%) and elevated bilirubin (5/26, 19.2\%). Rare adverse effects encompassed dizziness, nausea, and vomiting. Mild side effects were well tolerated and could be controlled by symptomatic treatment. Grade 3 adverse events included hypertension (3, 11.5\%), leukopenia (3,11.5\%), thrombocytopenia (2, 7.7\%), anemia (2, $3.8 \%)$, proteinuria $(1,3.8 \%)$, and elevated transaminase $(1,3.8 \%)$. None of the patients developed grade 4 toxicity. Adverse effects were manageable with symptomatic treatment or dose reduction, and there were no drug-related deaths. The initial dose of apatinib was $500 \mathrm{mg}$ taken once per day and adjusted according to the patients' intolerance. If serious adverse events occurred with intolerance in some cases, the dose was reduced to $250 \mathrm{mg}$ temporarily. Dose readjustments were made after the adverse reactions had been resolved and the patients had stabilized.

\section{DISCUSSION}

Cholangiocarcinoma is a kind of highly malignant tumor originating from the biliary epithelial cells. Depending on the 
A

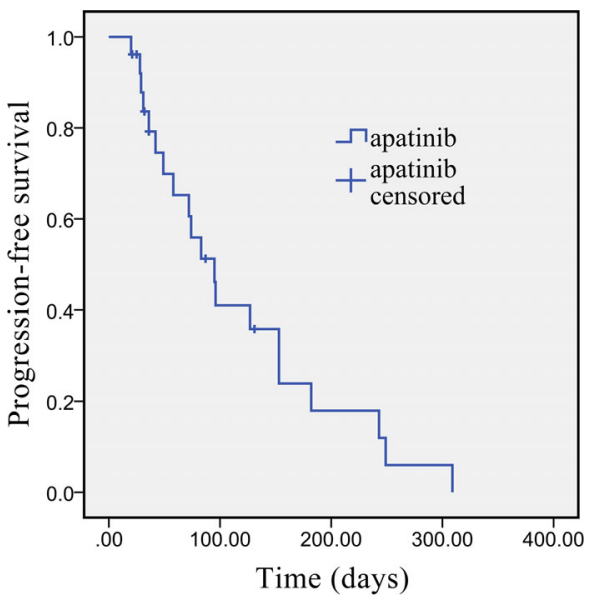

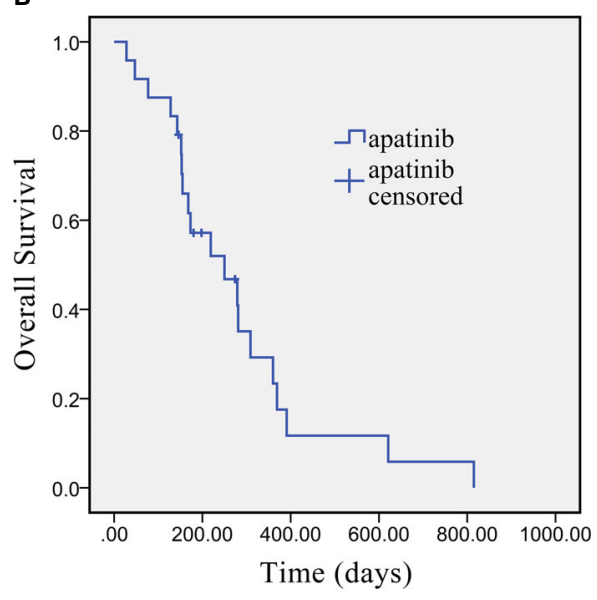

FIGURE 1 | Kaplan-Meier survival curve of PFS (A) and OS (B) in advanced cholangiocarcinoma patients who underwent apatinib monotherapy as the second-line treatment.

anatomic site, it is classified as intrahepatic and extrahepatic cholangiocarcinoma and gall bladder cancer. It is characterized by insensitivity to conventional chemotherapy and dismal prognosis with median OS of 12 months. In recent years, the morbidity rate of intrahepatic cholangiocarcinoma has shown a linear upward trend. Moreover, $60-70 \%$ of patients are at an advanced or very late stage at the time of diagnosis, and they are incurable due to few effective therapies available. Only $10 \%$ of patients are suitable to receive complete surgical resection, the only possible radical therapy, while all the remaining patients must receive palliative treatments.

Drugs approved by the FDA for non-resectable cholangiocarcinoma include gemcitabine, capecitabine, cisplatin, oxaliplatin, 5-fluorouracil, and combination therapy (15). Recently, systemic chemotherapies are considered the

TABLE 2 | The log rank analysis of factors affecting PFS.

\begin{tabular}{|c|c|c|c|c|}
\hline \multirow[t]{2}{*}{ Variable } & \multirow[t]{2}{*}{$\mathbf{N}$} & \multicolumn{3}{|c|}{ PFS } \\
\hline & & $95 \%$ & Cl & $\mathbf{P}$ \\
\hline Age (years) & & & & 0.010 \\
\hline$\leq 60$ & 16 & 127 & 64.3-189.7 & \\
\hline$>60$ & 8 & 49 & $2.8-95.2$ & \\
\hline Sex & & & & 0.238 \\
\hline Male & 13 & 95 & $75.5-114.5$ & \\
\hline Female & 11 & 74 & $68.2-79.8$ & \\
\hline CA199 (U/ml) & & & & 0.456 \\
\hline$\leq 37$ & 9 & 72 & $66.0-124.0$ & \\
\hline$>37$ & 15 & 95 & 7.0-137.0 & \\
\hline ECOG performance status & & & & 0.829 \\
\hline 0 & 8 & 127 & 50.0-204.0 & \\
\hline $1-2$ & 16 & 74 & $52.4-95.6$ & \\
\hline Anatomic location & & & & 0.008 \\
\hline Intrahepatic cholangiocarcinoma & 13 & 153 & $79.7-226.3$ & \\
\hline Extrahepatic cholangiocarcinoma & 11 & 72 & $21.3-122.7$ & \\
\hline
\end{tabular}

$N$, the number of patients; PFS, progression-free survival; Cl, confidence interval. primary palliative treatment but with controversial efficacy. Combination Cisplatin plus gemcitabine is an appropriate option for the standard first-line chemotherapy regimen of advanced biliary cancer, based on the findings of a phase III ABC-02 study, which produced the mOS from 8.1 months prolonged to 11.7 months (16). Moreover, gemcitabine and oxaliplatin combined with erlotinib, as another therapy option, prolonged median PFS (5.9 months, 95\% CI: 4.7-7.1) and hazard ratio $(\mathrm{HR})(0.73,95 \% \mathrm{CI}: 0.53-1.00)$, demonstrating

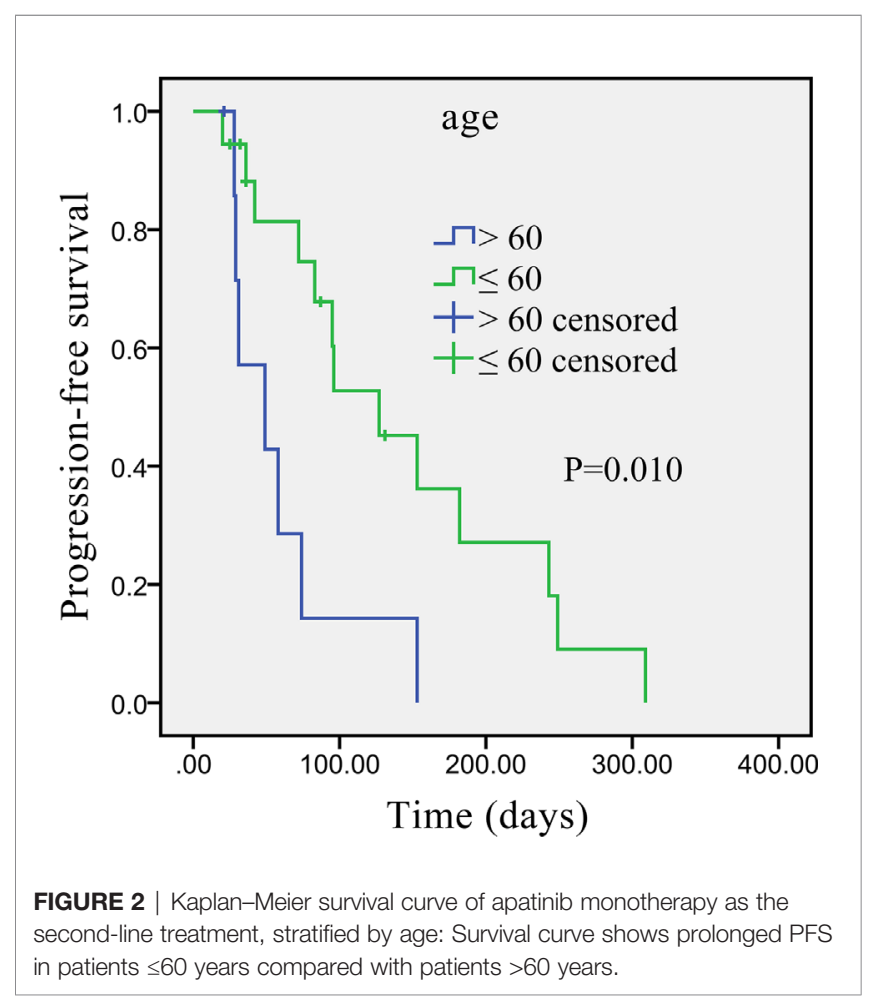




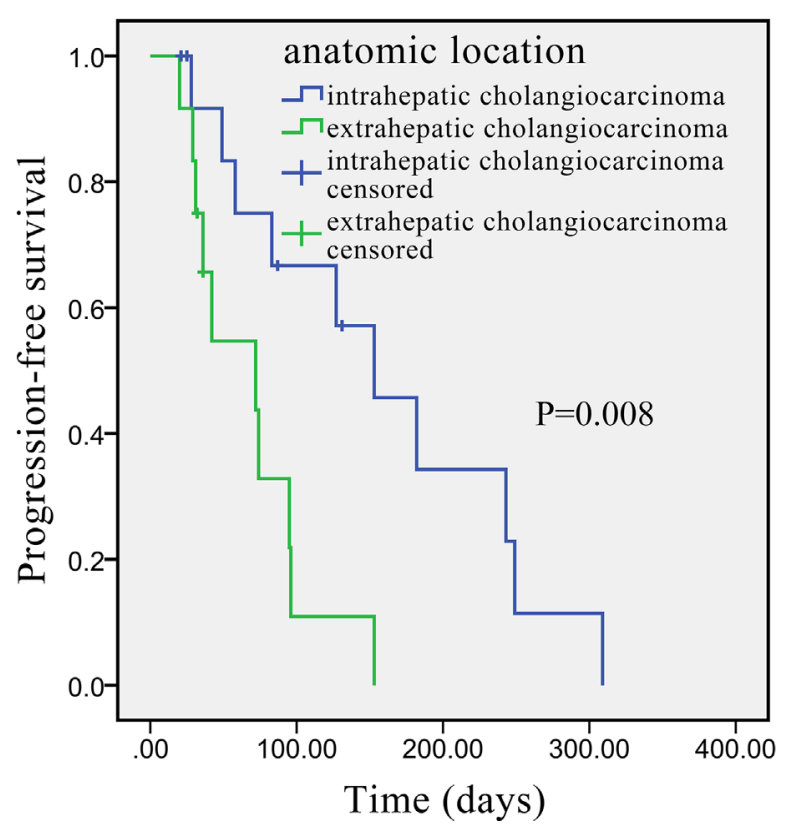

FIGURE 3 | Kaplan-Meier survival curve of apatinib monotherapy as the second-line treatment, stratified by anatomic location: Survival curve indicates that the PFS was higher in patients with intrahepatic than with extrahepatic cholangiocarcinoma.

improved efficacy, as shown by a multicentric, randomized, phase III clinical trial (17). Meanwhile, other chemotherapy regimens like gemcitabine combined with S-1 were also recommended as first-line options (18). A part of patients in whom the first-line treatment has failed are still in good physical condition; nevertheless, no clear recommendation for the second-line treatment is available, and little research has been conducted to solve this issue. Recently, emerging outputs from the multiple phase II trials demonstrated that fluoropyrimidine-based chemotherapy, considered as the second-line treatment, benefited patients with advanced

TABLE 3 | Main side effects of apatinib in the treatment of advanced cholangiocarcinoma.

\begin{tabular}{lcc}
\hline Main side effects & I-II, N (\%) & III-IV, N (\%) \\
\hline Secondary hypertension & $12(46.2)$ & $3(11.5)$ \\
Proteinuria & $11(42.3)$ & $1(3.8)$ \\
Thrombocytopenia & $16(61.5)$ & $2(7.7)$ \\
Leukocytopenia & $15(57.7)$ & $3(11.5)$ \\
Hand-foot syndrome & $10(38.5)$ & 0 \\
Anemia & $5(19.2)$ & $2(3.8)$ \\
Fatigue & $7(26.9)$ & 0 \\
ALT/AST increase & $6(23.1)$ & $1(3.8)$ \\
Serum bilirubin elevation & $5(19.2)$ & 0 \\
Anorexia & $6(23.1)$ & 0 \\
Oral mucositis & $3(11.5)$ & 0 \\
Rash & $1(3.8)$ & 0
\end{tabular}

$N$, the number of patients; \%, the percentage of patients. cholangiocarcinoma refractory to first-line chemotherapy (19). In recent ABC-06 trial, second-line mFOLFOX (folinic acid, 5-fluorouracil, and oxaliplatin) plus active symptom control (ASC) improved OS compared with ASC alone (12 months versus 6 months), providing evidence for the promising survival benefits of the use of second-line chemotherapy after progression on the cisplatin-gemcitabine combination (mFOLFOX+ASC versus ASC: HR 0.69, 95\% CI 0.50-0.97, $P=0.031)(20)$.

With such modest treatment outcomes and coming of the bottleneck stage for growing study of second-line therapy strategies, a better accurate understanding of tumor biology and the underlying disease mechanisms is vital for the selecting appropriate treatment, prediction of therapy outcomes. However, a series of staging systems and therapeutic and prognostic models for cholangiocarcinoma developed so far that most incorporated independent prognostic factors such as clinical parameters and histopathological features. Hence, the reality that a need to establish more precise and robust systems and models including clinical-pathological factors, molecular and genomic information, and tumor biomarkers predicts that cholangiocarcinoma therapy now entered in era of precision medicine. The widely utilized innovative techniques and highthroughput omics technologies have led to a number of novel targeted therapy drugs and biomarkers under investigation. Critically, the selection of the appropriate biomarker, comprehension of the tumor complex molecular mechanisms will guide us as to whether targeted therapy based on genetic changes will have a future in cholangiocarcinoma. The advent of genome-wide analyses using next-generation sequencing technologies have demonstrated the landscape of molecular mutations and identified several driver genetic alterations in bile duct cancer; for example, intracholangiocarcinoma have the highest of mutations in isocitrate dehydrogenase 1 (IDH1), and fibroblast growth factor receptor (FGFR) fusions which are of special interest, because they are not detectable in other liver malignancies, whereas the most prominent mutated gene extracholangiocarcinoma is BRAF (21). Pemigatinib, as the first targeted treatment for second-line strategy approved by FDA in 2020, showed clinically relevant potential of selective FGFR1-3 inhibitor for cholangiocarcinoma (22). The inhibition of the IDH1 mutation through its inhibitor ivosidenib represents a recent breakthrough in second-line therapy for cholangiocarcinoma, dramatically improved median PFS (2.7 months vs 1.4 months, HR $=0.37$, onesided $\mathrm{p}<0.0001)$ compared with placebo (23). As many of the targeted therapies have encouraging responses, critically, biomarker-driven clinical trials have to lay the groundwork for the best combinatorial approach of new drugs. Of note, successful approaches for targeting tumor angiogenesis have recently been worked out. Developmental angiogenesis is motivated through the interaction of VEGF and membrane receptor tyrosine kinases, including VEGFR-1, VEGFR-2, and VEGFR-3. Among those, VEGFR-2 is the most potent one involved in angiogenesis, and it supports vascular endothelial 
cells proliferation, migration, and survival via angiogenesismediated anti-apoptotic pathways, thus forming the basis for tumor progression and new lesions emergence (24). Consequently, many authors have suggested that reducing the overexpression of VEGF/VEGFR-2 would suppress tumor growth by prohibiting tumor angiogenesis (25). The introduction of anti-angiogenic drugs has proven to be investigated intensively as efficient subsequent options. In 2020, the first multicenter, randomized, placebo-controlled, phase II trial REACHIN reported that Regorafenib significantly increased median PFS (3.0 versus 1.5 months, $\mathrm{P}$ $=0.004)$ for unresectable cholangiocarcinoma in second- or subsequent-line setting, revealing that the angiogenesis drug regorafenib, as a multikinase inhibitor acting on VEGFR 1-3, platelet-derived growth factor receptor (PDGFR), FGFR, and other targets, showed good antitumor activity in biliary tumors (26). Moreover, Arkenau HT et al. used a combination of ramucirumab and pembrolizumab in 26 patients with pretreated advanced cholangiocarcinoma, suggested that the important role of the dual inhibition of the $\mathrm{PD}-1$ and VEGFR pathways, the median PFS around 1.5 months and an 11.3 months median OS (27). Although the regimens above showed underlying activity to some degree, they have been linked to a high incidence of severe adverse effects, relative lack of selectivity, as well as high cost.

A similar anti-angiogenic targeted drug, apatinib, suppressing tumor growth by highly and selectively inhibiting the tyrosine kinase activity of VEGFR-2, was approved by CFDA as early as in 2014 for advanced gastric or esophagogastric junction cancer following disease progression or recurrence after at least two previous systematic chemotherapy regimens (28). Furthermore, several phase II and III clinical trials have showcased that apatinib dramatically prolonged overall disease control rate and improved the clinical syndrome in several solid tumors, including advanced nonsmall cell lung cancer, breast cancer, hepatocellular carcinoma, and esophageal cancer (29-31). Overexpression of VEGF in intrahepatic cholangiocarcinoma is nearly 53\%, closely associated with a worse prognosis (32). As a novel inhibitor of tyrosine kinase targeting the intracellular ATP binding site of the receptor, apatinib is capable of downregulating the activity of the RAF/MEK/ERK and PI3K/Akt signaling pathways to block the VEGF/VEGFR-2 signal conduction. In that way, it promotes apoptosis and blocks proliferation and migration of vascular endothelial cells lines, thus decreasing microvessel density of tumor and inhibiting tumor growth (33). Considering its higher binding affinity to VEGFR-2 compared with other anti-angiogenic drugs, apatinib might be able to offer further potential therapeutic opportunities for treating advanced cholangiocarcinoma, especially for patients with high expression of VEGF (34). Actually, several studies had been reported to explore the role of apatinib. A prospective open-label phase II study (NCT03251443) indicated that apatinib as non-first-line therapy has promising anti-tumor activity, with ORR 11.5\%, DCR 50.0\%, and median PFS 2.0 months, for the pretreated advanced biliary tract cancers (35).
Likewise, in another study of second-line apatinib monotherapy, ORR was $10.0 \%$ (36).

Our study sought to prospectively analyze the application of apatinib monotherapy for advanced cholangiocarcinoma after gemcitabine-based treatment failure. We showed that the primary endpoint ORR occurred in 5 patients $(20.8 \%)$ and the secondary endpoint DCR in 15 patients $(62.5 \%)$. Median PFS and OS achieved 95 days (95\% CI: 79.70-154.34 days) and 250 days (95\% CI: 112.86-387.14 days), respectively. It is noteworthy that our treatment regimen was effective, with ORR in our study $(20.8 \%, 95 \%$ CI: 9.24-40.47\%) superior to the weighted mean ORR seen in previous studies $(7.7 \%, 95 \%$ CI: 4.6-10.9\%) according to a systematic review of Lamarca A et al. (14). The univariate analysis demonstrated significant effect of age and no significant effects of sex, ECOG performance status, and CA199 on the median PFS. Notably, we performed PFS based on the tumor site and noticed that patients with intrahepatic cholangiocarcinoma had prolonged PFS compared with patients with extrahepatic cholangiocarcinoma; thus, we speculated that apatinib monotherapy might be more beneficial for patients with intrahepatic cholangiocarcinoma. Although patients treated with apatinib achieved remarkable benefits, varied adverse effects cannot be ignored entirely during the application, including secondary hypertension, proteinuria, bone marrow suppression, hand-foot syndrome, and elevated transaminases. Most were classified as grade 1 or 2 , and only a minority of patients developed grade 3 toxicities, but most of them were gradually alleviated and clinically managed after dose adjustment with optimal supportive treatment.

A number of studies indicated that the dosage in various cancer subpopulations needs adjustments to improve efficacy and reduce the incidence of adverse reactions (37). When setting the dose at 750 or $850 \mathrm{mg}$ daily or $425 \mathrm{mg}$ twice a day in several previous studies, most patients experienced severe adverse effects. Apatinib was well-tolerated and showed significant efficacy at doses below $750 \mathrm{mg}$ per day in many solid tumors (38). Additionally, fewer AEs occurred in another study assessing apatinib for advanced cholangiocarcinoma, but similar findings have also been obtained for curative effect (rate of DCR $62.5 \%$ and the median PFS 95 days observed in our cohort versus the DCR rate $70.0 \%$ and the median PFS 3.0 months in that study). The difference in result might be explained by the fact that the dosage $(250 \mathrm{mg} /$ day $)$ in that study was lower than in our study (500 mg/day), which may have affected the treatment response (39). Thus far, there has been no more robust evidence for the effect of the dose of apatinib set at $500 \mathrm{mg}$ per day for the treatment of cholangiocarcinoma. Therefore, in the present study, we determined the initial dosage (500 $\mathrm{mg} /$ day) according to patients' tolerance and general status. Despite the reduction of dosage due to intolerant toxic effects, our study's curative effect was still maintained owing to patients' compliance. The result suggested that to balance dose and efficacy and AEs proper dosage regimen (a daily dose of $500 \mathrm{mg}$ is to start with, and subsequently decrease to $250 \mathrm{mg}$ per day gradually) might 
be able to exert superior therapeutic benefits and diminish the rate of severe AEs. Based on these findings, we hopefully provided a reference for the use of appropriate dose and medication cycle of apatinib as the second-line therapy for advanced cholangiocarcinoma.

Although major improvement has been achieved through modern molecular profiling, most results from clinical trials using targeting angiogenesis therapies have remained limited, thus highlighting the necessity to enhance a better understanding of tumor biology, and augment therapeutic potency by combining apatinib with other therapies (40). Transarterial chemoembolization (TACE) is a commonly used intra-arterial therapy for unresectable cholangiocarcinoma, which applies minimally invasive techniques to selectively insert a catheter into the artery supplying blood to the tumor and then injects chemotherapeutic drugs and embolic agents (41). In particular, chemotherapy achieves a high dose of cytotoxic payload and block tumor-feeding arteries by killing the tumor cells based on the cytotoxic effect, while at the same time, TACE causes a microenvironment in a state of ischemia and hypoxia in the embolized tissues, which further promotes tumor angiogenesis via higher expression of proangiogenic factors, such as VEGF (42). However, anti-angiogenic drugs block the growth of tumor vasculature and increase the cytotoxic drug concentration, and at a certain concentration, two therapies may have a synergistic rather than just additive effect. Accordingly, anti-tumor activity is very likely to be significantly enhanced in the combination of TACE treatments and apatinib in advanced cholangiocarcinoma therapy. One retrospective study in 35 cholangiocarcinoma patients provided evidence that the combination of apatinib and TACE has efficacy in improving survival profiles. Subgroup analysis indicated that both mOS (14.0 months versus 6.5 months, $\mathrm{P}=0.001, \chi 2=10.085)$ and mPFS (10.3 months versus 4.5 months, $\mathrm{P}=0.003, \chi 2=8.835)$ were prolonged in the apatinib plus TACE group compared with the apatinib alone, and further univariate Cox's regression analysis yielded that apatinib plus cTACE (vs. apatinib) were associated with increased mPFS (HR = $0.196, \mathrm{P}=0.004)$ and $\mathrm{mOS}(\mathrm{HR}=0.013, \mathrm{P}<0.001)$ (43). The introduction of immunotherapy altered the treatment regimens of various solid tumors, marking the era of modern cancer care (44, 45). A retrospective analysis suggested that the PD-1/PD-L1 pathway may be vital to the progression of unresectable intrahepatic cholangiocarcinoma in a cohort of 320 patients (45). In a combinatorial regimen randomized phase- 2 study of the PD-L1 inhibitor, atezolizumab, and the small molecule MEKinhibitor, cobimetinib in 77 patients with PD-L1 positive cholangiocarcinoma, the combination met its primary endpoint with a median PFS 3.65 months versus 1.87 months in the atezolizumab cohort (46). In this context, the anti-angiogenic therapy could be augmented by the immune checkpoint inhibitors by taking into account the established role of the immune system and hepatic microenvironment. Consequently, concurrent administration of apatinib with different therapy schemes as the standard practice is likely to be more therapeutically beneficial for advanced cholangiocarcinoma through the integrated control effect of systemic and locoregional therapy. In order to elucidate the synergistic efficacy and confirm the optimal mode of combination of apatinib, additional clinical studies are required in the future.

Notably, in another phase II clinical trial, for intermediate and advanced hepatocellular carcinoma patients, the patients receiving apatinib as the first-line therapy exhibited a tremendous potential in the long-term curative effect (mPFS: 8.7 months; mOS:13.8 months) (47). Therefore, we intend to conduct a new prospective study to fill the gap that there is a limited related theoretical basis for the therapeutic efficacy of apatinib in the first-line treatment of recurrent or metastatic advanced cholangiocarcinoma in the future.

There were a few limitations in our study. First, this study was a single-center trial, with the lack of a concurrent control arm. Second, the population of sample size was very small. Third, a portion of patients withdrew the consents, quit the trial before efficacy assessment, or was lost to follow-up, which might affect the real efficacy. Furthermore, we could not further analyze the clinical and molecular characteristics; this was limited due to the absence of appropriate biomarkers of anti-angiogenic agents. Therefore, large randomized controlled trials from multi-center with large sample sizes will include biomarkers investigation to predict the clinical value of apatinib in advanced cholangiocarcinoma.

\section{CONCLUSION}

Taken together, anti-angiogenesis plays an essential role in antitumor therapy. Improved efficiency of VEGFR blocking renders apatinib one of the practical and safe concurrent treatments in advanced cholangiocarcinoma detected initially, following failure of gemcitabine-based first-line chemotherapy. Further large-scale prospective and adequately powered clinical trials should be conducted to verify apatinib's effectiveness and safety as a secondline therapy, especially to explore more advantageous effects of combination therapies on cholangiocarcinoma and clarify the optimal therapeutic duration, dosage, clinical combination mode, and underlying mechanisms of apatinib regimen.

\section{DATA AVAILABILITY STATEMENT}

The raw data supporting the conclusions of this article will be made available by the authors, without undue reservation.

\section{ETHICS STATEMENT}

The studies involving human participants were reviewed and approved by First Affiliated Hospital of Zhengzhou University Ethic Committee (NO.SS-2018-08). The patients/participants provided their written informed consent to participate in this study. 


\section{AUTHOR CONTRIBUTIONS}

GZ collected the case data and drafted the manuscript; SG and $\mathrm{WH}$ were responsible for patient treatment and followup; GZ, LP and LH were involved in the acquisition of data and the search of the literature; WH made contributions to design, quality control of the study, and to critical revision of the manuscript. All authors contributed to the article and approved the submitted version. GZ and SG contributed equally in this study. Data curation: SG, LP, LH. Methodology: WH. Resources: GZ, SG. Writing - original draft: GZ. Writing - review and editing: GZ, WH.

\section{REFERENCES}

1. West J, Wood H, Logan R, Quinn M, Aithal GP. Trends in the Incidence of Primary Liver and Biliary Tract Cancers in England and Wales 1971-2001. Br J Cancer (2006) 94:1751-8. doi: 10.1038/sj.bjc.6603127

2. Malaguarnera G, Giordano M, Paladina I. Markers of Bile Duct Tumors. World J Gastrointest Oncol (2011) 3:49-59. doi: 10.4251/wjgo.v3.i4.49

3. Tamada K, Ushio J, Sugano K. Endoscopic Diagnosis of Extrahepatic Bile Duct Carcinoma: Advances and Current Limitations. World J Clin Oncol (2011) 2:203-16. doi: 10.5306/wjco.v2.i5.203

4. Brown, Kimberly M. Multidisciplinary Approach to Tumors of the Pancreas and Biliary Tree. Surg Clinics North America (2009) 89:115-31. doi: 10.1016/ j.suc.2008.09.022

5. Du H, Zhao J, Hai L, Wu J, Yi H, Shi Y. The Roles of Vasohibin and its Family Members: Beyond Angiogenesis Modulators. Cancer Biol Ther (2017) 18:82732. doi: 10.1080/15384047.2017.1373217

6. Ranieri G, Ammendola M, Marech I, Laterza A, Abbate I, Oakley C, et al. Vascular Endothelial Growth Factor and Tryptase Changes After Chemoembolization in Hepatocarcinoma Patients. World J Gastroenterol (2015) 21:6018-25. doi: 10.3748/wjg.v21.i19.6018

7. Zhao Y, Adjei AA. Targeting Angiogenesis in Cancer Therapy: Moving Beyond Vascular Endothelial Growth Factor. Oncologist (2015) 20:660-73. doi: 10.1634/theoncologist.2014-0465

8. Peng QX, Han YW, Zhang YL, Hu J, Wan Q. Apatinib Inhibits VEGFR-2 and Angiogenesis in an In Vivo Murine Model of Nasopharyngeal Carcinoma. Oncotarget (2017) 8:52813-22. doi: 10.18632/oncotarget. 17264

9. Haijun Z. Apatinib for Molecular Targeted Therapy in Tumor. Drug Des Devel Ther (2015) 9:6075-81. doi: 10.2147/DDDT.S97235

10. Tian S, Quan H, Xie C, Guo H, Lu F, Xu Y, et al. YN968D1 is a Novel and Selective Inhibitor of Vascular Endothelial Growth Factor Receptor-2 Tyrosine Kinase With Potent Activity In Vitro and In Vivo. Cancer Sci (2011) 102:1374-80. doi: 10.1111/j.1349-7006.2011.01939.x

11. Peng H, Zhang Q, Li J, Zhang N, Hua Y, Xu L, et al. Apatinib Inhibits Vegf Signaling and Promotes Apoptosis in Intrahepatic Cholangiocarcinoma. Oncotarget (2016) 7:17220-9. doi: 10.18632/oncotarget.7948

12. Huang J, Fan Q, Lu P, Ying J, Ma C, Liu W, et al. Icotinib in Patients With Pretreated Advanced Esophageal Squamous Cell Carcinoma With Egfr Overexpression or EGFR Gene Amplification: A Single-Arm, Multicenter Phase 2 Study. Thorac Oncol (2016) 11:910-7. doi: 10.1016/j.jtho.2016.02.020

13. Lu W, Jin X-L, Yang C, Du P, Jiang FQ, Ma JP, et al. Comparison of Efficacy Between TACE Combined With Apatinib and TACE Alone in the Treatment of Intermediate and Advanced Hepatocellular Carcinoma: A Single-Center Randomized Controlled Trial. Cancer Biol Ther (2017) 18:433-8. doi: 10.1080/15384047.2017.1323589

14. Lamarca A, Hubner RA, David Ryder W, Valle JW. Second-Line Chemotherapy in Advanced Biliary Cancer: A Systematic Review. Ann Oncol (2014) 25:2328-38. doi: 10.1093/annonc/mdu162

15. Subbiah IM, Subbiah V, Tsimberidou AM, Naing A, Kaseb AO, Javle M, et al. Targeted Therapy of Advanced Gallbladder Cancer and Cholangiocarcinoma

\section{FUNDING}

This work was funded by National Undergraduate Training Programs for Innovation and Entrepreneurship. Grant Number: 202010459182.

\section{ACKNOWLEDGMENTS}

We thank our colleagues from First Affiliated Hospital of Zhengzhou University for a supportive research environment and all patients and families for their enduring participation and their invaluable contribution.

With Aggressive Biology: Eliciting Early Response Signals From Phase I Trials. Oncotarget (2013) 4:153-62. doi: 10.18632/oncotarget.832

16. Valle J, Wasan H, Palmer DH, Cunningham D, Anthoney A, Maraveyas A, et al. Cisplatin Plus Gemcitabine Versus Gemcitabine for Biliary Tract Cancer. N Engl J Med (2010) 362:1273-81. doi: 10.1056/NEJMoa0908721

17. Lee J, Park SH, Chang HM, Kim JS, Choi HJ, Lee MA, et al. Gemcitabine and Oxaliplatin With or Without Erlotinib in Advanced Biliary-Tract Cancer: A Multicentre, Open-Label, Randomized, Phase 3 Study. Lancet Oncol (2012) 12:181-8. doi: 10.1016/S1470-2045(11)70301-1

18. Morizane C, Okusaka T, Mizusawa J, Katayama H, Ueno M, Ikeda M, et al. Combination Gemcitabine Plus S-1 Versus Gemcitabine Plus Cisplatin for Advanced/Recurrent Biliary Tract Cancer: The Fuga-Bt(Jcog1113) Randomized Phase III Clinical Trial. Ann Oncol (2019) 30:1950-8. doi: 10.1093/annonc/mdz402

19. Finn RS, Ryoo BY, Merle P, Kudo M, Bouattour M, Lim HY, et al. Pembrolizumab As Second-Line Therapy in Patients With Advanced Hepatocellular Carcinoma in KEYNOTE-240: A Randomized, Double-Blind, Phase III Trial. J Clin Oncol (2020) 38:193-202. doi: 10.1200/JCO.19.01307

20. Lamarca A, Palmer DH, Wasan HS, Ross PJ, Ma YT, Arora A, et al. Abc-06 | A Randomized Phase Iii, Multi-Center, Open-Label Study of Active Symptom Control (Asc) Alone or ASC With Oxaliplatin/5-FU Chemotherapy (Asc + mFOLFOX) for Patients (Pts) With Locally Advanced/Metastatic Biliary Tract Cancers (Abc) Previously-Treated With Cisplatin/Gemcitabine (CisGem) Chemotherapy. J Clin Oncol (2019) 37:4003. doi: 10.1016/S14702045(21)00027-9

21. Nakamura H, Arai Y, Totoki Y, Shirota T, ElZawahry A, Kato M, et al. Genomic Spectra of Biliary Tract Cancer. Nat Genet (2015) 47:1003-10. doi: 10.1038/ng.3375

22. Abou-Alfa GK, Sahai V, Hollebecque A, Vaccaro G, Melisi D, Al-Rajabi R, et al. Pemigatinib for Previously Treated, Locally Advanced or Metastatic Cholangiocarcinoma: A Multicentre, Open-Label, Phase 2 Study. Lancet Oncol (2020) 21:671-84. doi: 10.1016/S1470-2045(20)30109-1

23. Abou-Alfa GK, Macarulla T, Javle MM, Kelley RK, Lubner SJ, Adeva J, et al. Ivosidenib in IDH1-mutant, Chemotherapy-Refractory Cholangiocarcinoma (Claridhy): A Multicentre, Randomized, Double-Blind, Placebo-Controlled, Phase 3 Study. Lancet Oncol (2020) 21:796-807. doi: 10.1016/S1470-2045(20)30157-1

24. Oliveria RLD, Hamm A, Mazzone M. Growing Tumor Vessels: More Than One Way to Skin a Cat-Implications for Angiogensis Targeted Cancer Therapies. Mol Aspects Med (2011) 32:71-87. doi: 10.1016/j.mam.2011.04.001

25. Wang LY, Gong S, Gao LP, Hou LX, He W. Apatinib for Treating Advanced Intrahepatic Cholangiocarcinoma After Failed Chemotherapy: A Case Report and Literature Review. Med (Baltimore) (2018) 97:e13372. doi: 10.1097/ MD.0000000000013372

26. Demol A, Borbath I, Van den Eynde M, Houbiers G, Peeters M, Marechal R, et al. Regorafenib After Failure of Gemcitabine and Platinum-Based Chemotherapy for Locally Advanced/Metastatic Biliary Tumors: REACHIN, a Randomized, Double-Blind, Phase Ii Trial. Ann Oncol (2020) 9:31. doi: 10.1016/j.annonc.2020.05.018

27. Arkenau HT, Martin-Liberal J, Calvo E, Penel N, Krebs MG, Herbst RS, et al. Ramucirumab Plus Pembrolizumab in Patients With Previously Treated Advanced 
or Metastatic Biliary Tract Cancer: Nonrandomized, Open-Label, Phase I Trial (Jvdf). Oncology (2018) 23:1407. doi: 10.1634/theoncologist.2018-0044

28. Li J, Qin S, Xu J, Xiong J, Wu C, Bai Y, et al. Randomized, Double-Blind, Placebo-Controlled Phase Iii Trial of Apatinib in Patients With Chemotherapy-Refractory Advanced or Metastatic Adenocarcinoma of the Stomach or Gastroesophageal Junction. J Clin Oncol (2016) 34:1448-54. doi: 10.1200/JCO.2015.63.5995

29. Hu X, Cao J, Hu W, Wu C, Pan Y, Cai L, et al. Multicenter Phase Ii Study of Apatinib in non-Triple-Negative Metastatic Breast Cancer. BMC Cancer (2014) 14:820. doi: 10.1186/1471-2407-14-820

30. Liu Z, Ou W, Li N, Wang SY. Apatinib Monotherapy for Advanced non-Small Cell Lung Cancer After the Failure of Chemotherapy or Other Targeted Therapy. Thorac Cancer (2018) 9:1285-90. doi: 10.1111/1759-7714.12836

31. Aoyama T, Yoshikawa T. Targeted Therapy: Apatinib-New Third-Line Option for Refractory Gastric or GEJ Cancer. Nat Rev ClinOncol (2016) 13:268-70. doi: 10.1038/nrclinonc.2016.53

32. Huang M, Huang B, Li G, Zeng S. Apatinib Affect Vegf- Mediated Cell Proliferation, Migration, Invasion, Via Blocking VEGF2/RAF/MEK/ERK and PI3K/AKT Pathways in Cholangiocarcinoma Cell. BMC Gastroenterol (2018) 18:169. doi: 10.1186/s12876-018-0870-3

33. Sia D, Tovar V, Moeini A, LIovet JM. Intrahepatic Cholangiocarcinoma: Pathogenesis and Rationale for Molecular Therapie. Oncogene (2013) 32:4861-70. doi: 10.1038/onc.2012.617

34. Peng S, Zhang Y, Peng H, Ke Z, Xu L, Su T, et al. Intracellular Autocrine Vegf Signaling Promotes EBDC Cell Proliferation, Which can be Inhibited by Apatinib. Cancer Lett (2016) 373:193-202. doi: 10.1016/j.canlet.2016.01.015

35. Mao JZ, Yang X, Lin JZ, Yang XB, Wang DX, Zhang L. Apatinib as non-FirstLine Treatment in Patients With Intrahepatic Cholangiocarcinoma. J Cancer (2021) 12:1555-62. doi: 10.7150/jca.53482

36. Lin GH, Wang BC, Wu XW, Sun T, Chen LL, Lu CL, et al. Efficacy and Safety of Apatinib Treatment for Patients With Advanced Intrahepatic Cholangiocarcinoma. Cancer Manag Res (2020) 12:11523-6. doi: 10.2147/ CMAR.S257526

37. Yu M, Gao Z, Dai X, Gong H, Zhang L, Chen X, et al. Population Pharmacokinetic and Covariate Analysis of Apatinib, an Oral Tyrosine Kinase Inhibitor, in Healthy Volunteers and Patients With Solid Tumors. Clin Pharmacokinet (2017) 56:65-76. doi: 10.1007/s40262-016-0427-y

38. Hu X, Zhang J, Xu B, Jiang Z, Ragaz J, Tong Z, et al. Multicenter Phase Ii Study of Apatinib, a Novel Vegfr Inhibitor in Heavily Pretreated Patients With Metastatic Triple-Negative Breast Cancer. Int J Cancer (2014) 135:1961-9. doi: $10.1002 / \mathrm{ijc} .28829$

39. Lin G, Wang B, Wu X, Sun T, Chen L, Lu C, et al. Efficacy and Safety of Apatinib Treatment for Patients With Advanced Intrahepatic
Cholangiocarcinoma. Cancer Manag Res (2020) 12:11523-6. doi: 10.2147/ CMAR.S257526

40. Simile MM, Bagella P, Vidili G, Spanu A, Manetti R, Seddaiu MA, et al. Targeted Therapies in Cholangiocarcinoma: Emerging Evidence From Clinical Trials. Medicina (2019) 55:42. doi: 10.3390/medicina55020042

41. Avic LJ, Chapiro J, Geschwind JH. Intra-Arterial Embolotherapy for Intrahepatic Cholangiocarcinoma: Update and Future Prospects. Hepatobiliary Surg Nutr (2017) 6:7-21. doi: 10.21037/hbsn.2016.11.02

42. Schicho A, Hellerbrand C, Kruger K, Beyer LP, Wohlgemuth W, Niessen C, et al. Impact of Different Embolic Agents for Transarterial Chemoembolization (Tace) Procedures on Systemic Vascular Endothelial Growth Factor (Vegf) Levels. J Clin Transl Hepatol (2016) 4:288-92. doi: 10.14218/JCTH.2016.00058

43. Hu Y, Hao M, Chen Q, Chen Z, Lin H. Comparison of the Efficacy and Safety Among Apatinib Plus Drug-Eluting Bead Transarterial Chemoembolization (Tace), Apatinib Plus Conventional TACE and Apatinib Alone in Advanced Intrahepatic Cholangiocarcinoma. Am J Transl Res (2020) 12:6584-98.

44. Mollica V, Di Nunno V, Gatto L, Santoni M, Cimadamore A, Cheng L, et al. Novel Therapeutic Approaches and Targets Currently Under Evaluation for Renal Cell Carcinoma: Waiting for the Revolution. Clin Drug Invest (2019) 39:503-19. doi: 10.1007/s40261-019-00773-w

45. Modena A, Ciccarese C, Iacovelli R, Brunelli M, Montironi R, Fiorentino M, et al. Immune Checkpoint Inhibitors and Prostate Cancer: A New Frontier? Oncol Rev (2016) 10:293. doi: 10.4081/oncol.2016.293

46. Yarchoan M, Cope L, Anders RA, Noonan A, Goff LW, Goyal L, et al. Abstract CT043: A Multicenter Randomized Phase 2 Trial of Atezolizumab as Monotherapy or in Combination With Cobimetinib in Biliary Tract Cancers (Btcs): A Nci Experimental Therapeutics Clinical Trials Network (Etctn) Study. AACR (2020) Session VCTPL04, CT043: 80.

47. Hou Z, Zhu K, Yang X, Chen P, Zhang W, Cui Y. Apatinib as First-Line Treatment in Patients With Advanced Hepatocellular Carcinoma: A Phase II Clinical Trial. Ann Oncol (2020) 8:1047-7. doi: 10.21037/atm-20-2990

Conflict of Interest: The authors declare that the research was conducted in the absence of any commercial or financial relationships that could be construed as a potential conflict of interest.

Copyright (c) 2021 Zhang, Gong, Pang, Hou and He. This is an open-access article distributed under the terms of the Creative Commons Attribution License (CC BY). The use, distribution or reproduction in other forums is permitted, provided the original author(s) and the copyright owner(s) are credited and that the original publication in this journal is cited, in accordance with accepted academic practice. No use, distribution or reproduction is permitted which does not comply with these terms. 